\title{
Production of Ball-Lightning-Like Luminous Balls by Electrical Discharges in Silicon
}

\author{
Gerson Silva Paiva and Antonio Carlos Pavão \\ Departamento de Química Fundamental, Universidade Federal de Pernambuco, 50740-540, Recife, Pernambuco, Brazil \\ Elder Alpes de Vasconcelos* \\ Centro Acadêmico do Agreste, Universidade Federal de Pernambuco, 55002-970, Caruaru, Pernambuco, Brazil \\ Odim Mendes, Jr. \\ Departamento de Geofísica Espacial, Instituto Nacional de Pesquisas Espaciais, 12245-970, São José dos Campos, São Paulo, Brazil \\ Eronides Felisberto da Silva, Jr. \\ Departamento de Física, Universidade Federal de Pernambuco, 50670-901, Recife, Pernambuco, Brazil
}

(Received 10 June 2006; published 24 January 2007)

\begin{abstract}
We performed electric arc discharges in pure Si to generate luminous balls with lifetime in the order of seconds and several properties usually reported for natural ball lightning. This simple experiment does not rely on energy sources and excitation mechanisms that are improbable in the natural phenomenon and clearly demonstrates the role of vaporization and oxidation of $\mathrm{Si}$, as proposed by the Abrahamson-Dinniss theory for ball-lightning formation.
\end{abstract}

DOI: 10.1103/PhysRevLett.98.048501

PACS numbers: $92.60 . P w, 52.80 . \mathrm{Mg}$

Introduction.-The properties attributed to the balllightning phenomenon have been deduced from reports collected from hundreds of witnesses in the past two centuries [1-13]. No existing theory or model can account for all (and sometimes contradictory) observations of ball lightning. Different mechanisms able to generate luminous balls with similar appearance can be imagined and this gave rise to a variety of theories for the origin of ball lightning. A recent theory that has attracted great attention was proposed by Abrahamson and Dinniss [5-8]. They proposed that ball lightning is due to oxidation of Si nanoparticles in the atmosphere. The $\mathrm{Si}$ nanoparticles are formed as a result of the reaction of $\mathrm{Si}$ oxides and carbon in the soil. At the high temperatures created by the lightning strike, the carbon in the soil chemically reduces the $\mathrm{Si}$ oxides to the vaporized, metallic form of $\mathrm{Si}: \mathrm{SiO}_{2}+2 \mathrm{C} \rightarrow$ $\mathrm{Si}+2 \mathrm{CO}$. As the hot vapor cools in the atmosphere, the $\mathrm{Si}$ condenses into an aerosol of nanometer-sized Si particles in the air. Electrical charges created in the heat gather around the surface of aerosol, binding it together and the resulting ball begins to glow with the heat of the $\mathrm{Si}$ oxidation in the atmosphere: $\mathrm{Si}+\mathrm{O}_{2} \rightarrow \mathrm{SiO}_{2}$. This model has been extended by Abrahamson to include a broad range of starting materials: from soil, or soil and wood, to also include metal and plastic or metal and wood [8].

Laboratory experiments have been aimed at testing one particular mechanism of ball formation. For instance, Barry tested the combustion mechanism and observed fireballs by triggering discharges in atmospheres containing small amounts of hydrocarbons [14]. Silberg [15] and Golka, Jr. [16] obtained luminous balls by switching high currents through metal electrodes. Recently, fireballs have been obtained using microwave excitation [17,18].
However, fireballs obtained by microwaves have short lifetime after the microwave source is removed and it is not likely that in the natural phenomenon there is a supply of microwave energy appropriate for production of fireballs with the long lifetimes observed (seconds or tens of seconds). Abrahamson tested his model by means of high voltage electrical discharges into soil samples, but none of the tests produced luminous balls [8]. In this work, we tested Abrahamson's theory by vaporizing, at normal atmospheric pressure, small pieces of highly pure Si wafers by an electric arc. The arc generated by the interruption of the electric circuit results in very high temperatures, therefore melting or vaporizing locally the Si pieces. We were able to generate luminous balls that have long lifetimes and several of the properties usually reported for natural ball lightning.

Experimental details. - We used pieces of 2-inch diameter, (111) or (100), 0.02 to $1 \Omega \mathrm{cm}$ resistivity, $p$-type doped, $350 \mu \mathrm{m} \pm 50 \mu \mathrm{m}$ thick Si wafers placed on a $5 \mathrm{~mm}$ thick $1000 \times 1000 \mathrm{~mm}^{2}$ flat steel plate as the base electrode and a tungsten (or graphite) top electrode, as shown in Fig. 1(a). The top electrode is $4 \mathrm{~mm}$ in diameter and $30 \mathrm{~cm}$ long. The voltage at the secondary is in the range from 20 to $25 \mathrm{~V}$ and the current varies from 100 to $140 \mathrm{~A}$. The top electrode is movable and is hand-operated. The operator gently touches the Si piece with the top electrode and closes the circuit, as shown in Fig. 1(b). Then, the top electrode is raised up to a distance of 1 to $2 \mathrm{~mm}$, approximately. An electrical arc is formed during the upraising movement, as shown in Fig. 1(c). During the upraising movement of the top electrode, hot-glowing fragments and, eventually, the ball-lightning-like luminous balls fly away in all directions. The arc is then extinguished 


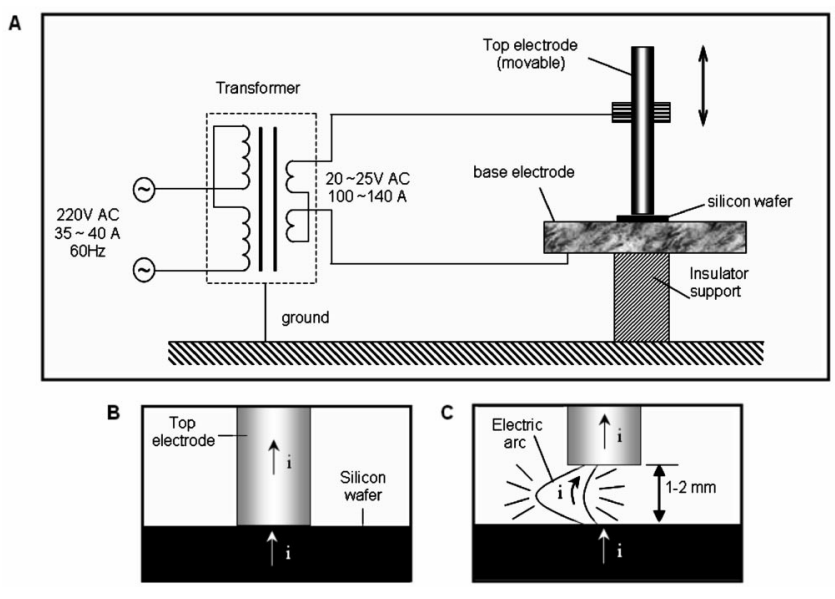

FIG. 1. (a) Experimental arrangement showing the power supply, electrode geometry and the Si wafer. (b) The top electrode is lowered until it touches the $\mathrm{Si}$ piece and closes the circuit. (c) The top electrode is raised up to a distance of 1 to $2 \mathrm{~mm}$, approximately. An electrical arc is formed during this upraising movement. Hot-glowing fragments and, eventually, ball-lightning-like luminous balls fly away in all directions.

by moving the top electrode farther from the base electrode. This whole procedure should take 1 to $2 \mathrm{sec}$, approximately. We filmed the experiments with a Sony TRV130 digital camera. We observed the surface of the $\mathrm{Si}$ pieces subjected to discharges by scanning electron microscopy (SEM) after discharge using a JEOL JSM5900. These surfaces were also scratched and the residues were analyzed in a Fourier Transform Infrared (FT-IR) system operating in transmission mode to determine the composition of the chains of particles observed in the SEM images. The discharges were performed at $29^{\circ} \mathrm{C}$ room temperature and a relative humidity of $70 \%$.

Results and discussion. - Video clips (supplementary videos 1-6) illustrating the experiment and the features of the balls are available [19]. The supplementary video 1 shows discharges which did not produce luminous balls. The supplementary videos 2-6 show discharges which produced luminous balls. Figures 2-4 consist in video frames selected from supplementary videos 2-4. Many small, hot-glowing fragments fly away in all directions during the discharge. It is clear that the luminous balls
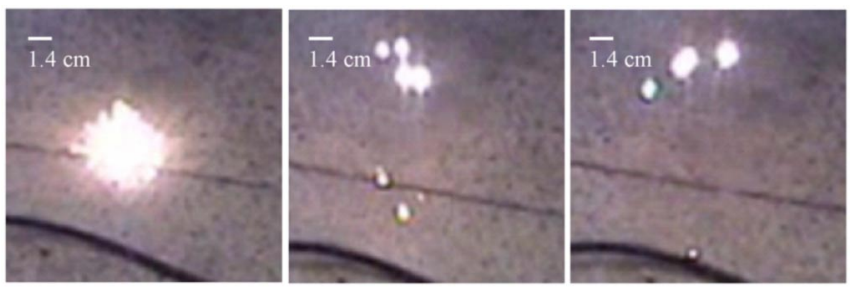

FIG. 2 (color online). Successive video frames showing the luminous balls bouncing off the ground. Time interval between the frames is $80 \mathrm{~ms}$. See also the supplementary video 2 [19].
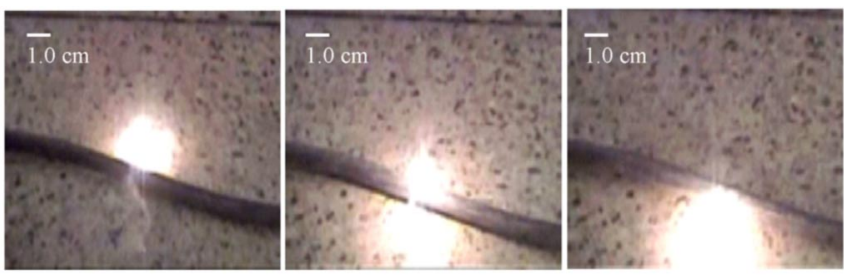

FIG. 3 (color online). Successive video frames showing a luminous ball passing through a small gap under an electrical conductor. Time interval between the frames is $80 \mathrm{~ms}$. See also the supplementary video 3 [19].

resembling the ball-lightning phenomenon have a very distinct behavior. Their apparent diameter is in the range from 1-4 cm, much bigger than a typical fragment. Also, their lifetime may be up to $8 \mathrm{~s}$, whereas the fragments cool very quickly, in $1 \mathrm{~s}$ approximately. Moreover, the luminous balls have properties that are similar to the properties of the balls observed in the natural phenomenon. Table I shows a comparison of the properties of luminous balls in this experiment with the properties observed in natural ball lightning $[9,11]$. We prepared Table I based on Ref. [9], which contains two collections of observations from different sources, namely, collection 1 and collection 2 . Observations in collection 1 were reported by correspondence to the authors of [9] themselves, whereas the observations in collection 2 were organized by the Russian BallLightning Committee. The convention in Table I is: (1c) stands for observation (c) from collection (1), (2c) means observation (c) from collection (2), and so on.

Figure 2 (from supplementary video 2) shows that the ball bounces off and moves erratically on the ground, sometimes breaking up in two or more smaller balls. The glowing balls sometimes jump to an height between 5 and $20 \mathrm{~cm}$. Their estimated velocity is in a range from 5 to $30 \mathrm{~cm} / \mathrm{s}$. Figure 3 (from supplementary video 3) shows that one of these glowing balls passed through a small gap under an electrical conductor, squeezing through a space smaller than its apparent diameter. This behavior can be related with the ability of the natural ball lightning to penetrate through little gaps, according to several eyewitnesses [9]. The balls leave smoke trails above it. The balls seem to be spinning because the smoke trails tend to form a
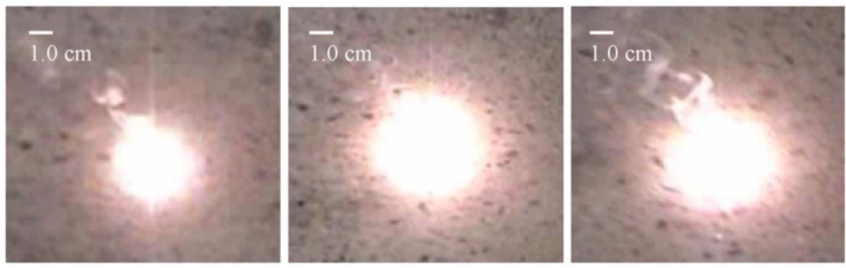

FIG. 4 (color online). Successive video frames showing a luminous ball leaving spiraling smoke trails above it. Time interval between the frames is $80 \mathrm{~ms}$. See also supplementary video 4 [19]. 
TABLE I. Comparison between artificial balls and natural balls.

\begin{tabular}{|c|c|}
\hline Property of the artificial luminous balls in this experiment & Eyewitness report describing similar property in natural balls ${ }^{\mathrm{a}}$ \\
\hline $\begin{array}{l}\text { To move over an extended, erratic path, } \\
\text { sometimes with varying speeds }\end{array}$ & $1 \mathrm{c} 1 \mathrm{~g} 1 \mathrm{n} 2 \mathrm{u}$ \\
\hline To subdivide into smaller balls & $2 \mathrm{c} 2 \mathrm{~h}$ \\
\hline Vibrating surface, sparks, "fluff-cotton-wool" appearance & $\lg 1 \mathrm{j} 2 \mathrm{e} 2 \mathrm{l} 2 \mathrm{n} 2 \mathrm{o} 2 \mathrm{u}$ \\
\hline To roll, to bounce off ground or solid objects & 1c $1 \mathrm{~g} 11$ 1p $1 \mathrm{f} 2 \mathrm{j} 2 \mathrm{~h} 2 \mathrm{q}$ \\
\hline To squeeze into confined spaces & $1 \mathrm{j} 2 \mathrm{~d} 2 \mathrm{p} 2 \mathrm{q} 2 \mathrm{~s}$ \\
\hline To spin & $\lg \ln 1 \mathrm{r}$ \\
\hline To burn objects upon contact & $1 \mathrm{i}$ \\
\hline Bright bluish-white or orange-white color & 1i $111 \mathrm{~m} 1 \mathrm{o} 2 \mathrm{k} 2 \mathrm{p} 2 \mathrm{u}$ \\
\hline Spherical in shape without well defined boundaries & $2 \mathrm{j} 2 \mathrm{n} 2 \mathrm{p} 2 \mathrm{q} 2 \mathrm{r} 2 \mathrm{~s}$ \\
\hline Lifetime 2 to 5 seconds & $1 \mathrm{j} 2 \mathrm{j} 2 \mathrm{k}$ \\
\hline
\end{tabular}

${ }^{a}$ Convention: $1 \mathrm{c}$ — collection (1), observation (c). 2c-collection (2), observation (c). Collections included in Ref. [9], as explained in the text.

spiral, as shown in Fig. 4 (from supplementary video 4). Also, inclination changes in this rotational axis (vertical to horizontal, for instance) may be responsible for the sudden increase in speed and changes in direction observed in their movement, as can be noticed in supplementary video 3 . The luminous balls behave like a jumping, elastic ball with glowing jets off its turbulent surface that apparently impulse it forward or sideways. These balls are hot (they burned polystyrene Styrofoam upon contact and ignited ethanol-imbibed cotton) and decay leaving no trace.

The SEM images of the surface of control samples not exposed to electrical discharges is regular and without holes or particles [Fig. 5(a)]. On the other hand, the surface of the samples subjected to electrical discharges, shows holes [Figs. 5(b) and 5(c)] and chains of micrometer-sized particles [Fig. 5(d)]. The holes indicate that the discharge has penetrated beneath the wafer surface and caused melting or vaporization of the material. Lightning strikes have been known to create glassy walled, hollow tubes just under the ground surface. The tubes, known as fulgurites, form where lightning melts and vaporizes soil along their paths. Some researchers have suggested that materials from such cavities might play a role in ball lightning. Almost three decades ago, Andrianov and Sinitsyn [20] proposed that the glowing material of ball lightning is generated during the formation of fulgurites. The FT-IR spectrum of the particles shown in Fig. 5(d) had a strong absorption band in the $1103 \mathrm{~cm}^{-1}$ region (Si-O stretching), thus confirming that these particles are composed by $\mathrm{SiO}_{2}$, probably formed by the oxidation of the vaporized or molten Si.

In summary, this experiment has four important features: (1) It does not rely on energy sources and excitation mechanisms which are improbable in the natural phenomenon. (2) It clearly demonstrates the role played by the vaporization and oxidation of $\mathrm{Si}$, as proposed by the Abrahamson-Dinniss theory for ball-lightning formation. (3) It generates luminous balls with long lifetimes (up to $8 \mathrm{sec}$ ) and several properties observed in the natural phenomenon (10 properties, at least), and (4) it is very simple.

We would like to discuss two limitations of this experiment. First, the production of the luminous balls is not under complete control. Second, free-floating balls were not observed. The lack of precise control of the production of the luminous balls is a common characteristic shared by many ball-lightning experiments. This reflects our limited understanding of the various chemical and physical processes triggered by the discharges. For example, Bychkov reports that a red-white ball resembling ball lightning appeared after 450-500 trial discharges during an experiment involving glass and wood [21]. In our experiments, we observed that the temperature and movements of the
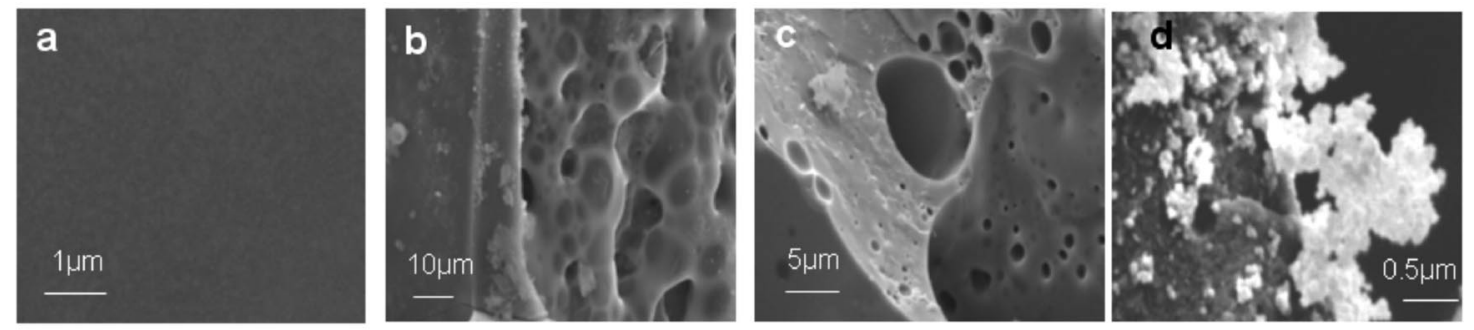

FIG. 5. SEM of the Si wafer before (a) and after electrical discharge (b),(c),(d). The surface of the samples subjected to electrical discharges shows holes (b),(c) and chains of micrometer-sized particles (d). 
top electrode are somehow related with the production of the luminous balls. The balls only appear after the top electrode is glowing hot. The movements of the top electrode also influence. The top electrode is movable and is hand operated. The operator touches the wafer closing the circuit, and then raises the top electrode up to a distance of 1 to $2 \mathrm{~mm}$, approximately. An electrical arc is formed when the contact is broken during the upraising movement of the top electrode. Hot-glowing fragments and, eventually, the ball-lightning-like luminous balls fly away. The arc is then extinguished by moving the top electrode farther from the base electrode. This whole procedure should take 1 to 2 seconds, approximately. If the up and down movements of the top electrode are too fast or too slow, the luminous balls do not appear. In order to improve the reproducibility of the up and down movements of the top electrode, one can use some kind of electrically-driven mechanical arm. In fact, we made some preliminary studies with the top electrode connected to a relay armature and observed improved reproducibility in the production of luminous balls. Our statistics (with the hand-operated top electrode) is approximately 1 event out of 30 trials.

Free-floating balls were not generated by our experiments. In the context of Abrahamson's theory, ball lightning is a network of nanoparticles being oxidized by the surroundings. It is reasonable to admit that such a network may have different density depending on particle size, network morphology, etc. In addition, external forces generated by electric fields or by the wind, for example, may change its buoyancy. Possibly, the oxidizing networks generated in our experiments are too dense to float. We are currently investigating the effects of the external forces, as well as changes in discharge energy and materials to better understand this issue.

To compare these results with those using a non-Si electrode, we performed similar experiments replacing the $\mathrm{Si}$ wafers by: (1) $\mathrm{Al}$ and $\mathrm{Cu}$ foils, (2) salty water, (3) dry and wet $\mathrm{SiO}_{2}$ microspheres (the type used for sand blasting), (4) moistened wood and (5) a mixture of moistened wood and $\mathrm{SiO}_{2}$ microspheres. The conductivity values of these materials are very different from the $\mathrm{Si}$ wafers, so we subjected each one of these materials to various combinations of voltage and current to ensure that we explored a reasonably wide range of discharge powers. None of these materials produced luminous balls with the peculiar behavior observed when the pieces of $\mathrm{Si}$ wafers are used.

Conclusions. - Low voltage electric arc discharges in pure Si can generate luminous balls with several of the properties usually reported for natural ball lightning. We discussed the main advantages and limitations of this experiment in the framework of Abrahamson-Dinniss theory of ball lightning. We propose that the use of pure $\mathrm{Si}$ wafers probably optimized the evaporation of $\mathrm{Si}$ and favored the formation of the oxidizing Si nanoparticles networks predicted by the theory, so that ball-lightning-like luminous balls could appear associated with discharges involving currents much lower than expected in normal lightning strikes.

The authors are grateful to Giovanni R.R. Barros (Departamento de Engenharia Elétrica e Sistemas de Potência-Universidade Federal de Pernambuco) and Wellington Lucena for their comments and help during the experiments, Eliete Barros by infrared analysis, and Francisco Rangel for microscope investigation. This work is supported by the Brazilian agencies CAPES and CNPq.

*Corresponding author.

[1] S. Singer, Nature (London) 198, 745 (1963).

[2] S. Singer, Nature (London) 350, 108 (1991).

[3] S. Singer, Phil. Trans. R. Soc. A 360, 5 (2002).

[4] D. J. Turner, Phil. Trans. R. Soc. A 360, 107 (2002).

[5] J. Abrahamson and J. Dinniss, Nature (London) 403, 519 (2000).

[6] J. Abrahamson, Phys. World 15, 22 (2002).

[7] R. Matthews, New Sci. 2233, 23 (2000).

[8] J. Abrahamson, Phil. Trans. R. Soc. A 360, 61 (2002).

[9] J. Abrahamson, A. V. Bychkov, and V.L. Bychkov, Phil. Trans. R. Soc. A 360, 11 (2002).

[10] M. A. Uman, Lightning (Dover, New York, 1969), Appendix C.

[11] M. Stenhoff, Ball Lightning: An Unsolved Problem in Atmospheric Physics (Springer-Verlag, New York, 1999).

[12] J. D. Barry, Ball Lightning and Bead Lightning: Extreme Forms of Atmospheric Electricity (Kluwer Academic, Dordrecht, 1980).

[13] V.A. Rakov and M. A. Uman, Lightning: Physics and Effects (Cambridge University Press, Cambridge, England, 2003), Chap. 20.

[14] J. D. Barry, J. Atmos. Terr. Phys. 30, 313 (1968).

[15] P. A. Silberg, J. Geophys. Res. 67, 4941 (1962).

[16] R. K. Golka, Jr., J. Geophys. Res. 99, 10679 (1994).

[17] Y. H. Ohtsuki and H. Ofuruton, Nature (London) 350, 139 (1991).

[18] V. Dikhtyar and E. Jerby, Phys. Rev. Lett. 96, 045002 (2006).

[19] See EPAPS Document No. E-PRLTAO-98-047705 for video clips showing the experiment and the luminous balls. For more information on EPAPS, see http://www. aip.org/pubservs/epaps.html.

[20] A. M. Andrianov and V. I. Sinitsyn, Sov. Phys. Tech. Phys. 22, 1342 (1977).

[21] V. L. Bychkov, Phil. Trans. R. Soc. A 360, 37 (2002). 